\title{
Frequency Response Simulation Analysis of Waveguide Photodetectors
}

\author{
João Paulo N. Torres ${ }^{1} \&$ Jorge Pereira ${ }^{1}$ \\ ${ }^{1}$ Instituto de Telecomunicações, DEEC/ Instituto Superior Técnico, Universidade de Lisboa, Lisboa, Portugal \\ Correspondence: João Paulo N. Torres, nstituto de Telecomunicações, DEEC/ Instituto Superior Técnico, \\ Universidade de Lisboa, Lisboa, Portugal. E-mail: joaotorres@tecnico.ulisboa.pt
}

Received: April 26, 2017

Accepted: May 3, 2017

Online Published: July 18, 2017

doi:10.5539/apr.v9n4p1

URL: https://doi.org/10.5539/apr.v9n4p1

\begin{abstract}
In waveguide photodetectors the bandwidth and the quantum efficiency may be specified almost independently because the absorption of light is in the longitudinal direction whereas the current flows in the transversal direction. Then, these devices may combine high bandwidth with high quantum efficiency. In this paper a detailed numerical analysis of the frequency response of waveguide photodetectors is presented. The calculations show that the capacitive effects limit the bandwidth but that by an appropriate choice of a series inductance the maximum bandwidth almost doubles.
\end{abstract}

Keywords: photodetector, waveguide photodetector, frequency response, modeling

\section{Introduction}

Optical communication systems rely on photodetectors characterized by high bandwidth and high quantum efficiency. The bandwidth determines the bit-rate of the optical communication system whereas the quantum efficiency is related to the detector's sensitivity.

The most important and basic photodetector device is the double heterojunction semiconductor pin photodiode (Bowers \& Burrus, 1987). For optical communication systems, working in the 1.3-1.6 $\mu \mathrm{m}$ range, the detector's absorption region is usually the ternary $\operatorname{In}_{0.53} \mathrm{Ga}_{0.47} \mathrm{As}$ semiconductor and the $\mathrm{n}$ and $\mathrm{p}$ contacts are of lattice matched InP (Pearsall, 1980). In the conventional pin photodetectors the bandwidth and quantum efficiency cannot be optimized simultaneously. In fact by decreasing the absorption region length there may be an increase of the bandwidth but the quantum efficiency decreases leading to a product bandwidth $\times$ quantum efficiency which is nearly constant for a wide range of absorption region lengths, taking values of the order of tens of $\mathrm{GHz}$ (Fernandes \& Pereira, 2011).

The independent control of the bandwidth and quantum efficiency may be obtained if the illumination is in the direction perpendicular to the current. In this way the transversal and longitudinal dimensions may be adjusted independently, i.e., the bandwidth and the quantum efficiency. The first photodetectors based on this concept were named Waveguide Photodetector (WGPD) where the waveguide itself is the absorption layer of the photodetector (Kato et al., 1992). For this device, by increasing the length of the absorption region and decreasing its thickness, it is possible to obtain high quantum efficiency and high bandwidth.

This paper investigates the frequency response of pin WGPD. The capacitive and inductive effects are included in the model. It is shown that the inductive effects may cancel in part the capacitive effects which lead to an increase of the photodetector's bandwidth.

\section{Structure}

The structure under study is represented schematically in Figure 1. The absorption region is of $\operatorname{In}_{0.53} \mathrm{Ga}_{0.47} \mathrm{As}$ and the $\mathrm{p}$ and $\mathrm{n}$ regions are of InP. Light is incident from the left and is absorbed in the ternary semiconductor along the longitudinal direction. The metallic contacts are deposited on the top of the InP regions and the device is reverse biased in the transversal direction. Without illumination the absorption region is depleted of carriers and the electrical field in this region is approximately constant. The electron-hole pairs generated by the illumination, when subjected to the electric field, travel in opposite directions to the contacts and a current may be established in the transversal direction. 


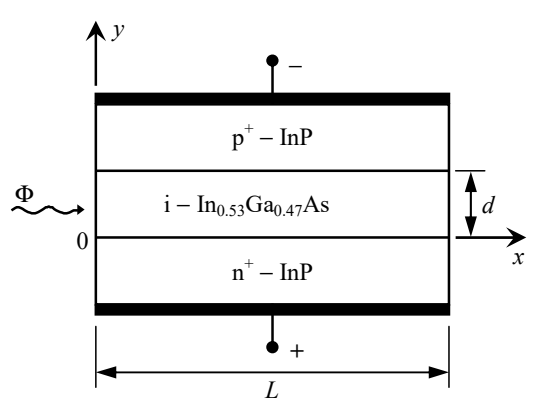

Figure 1. Schematics of the waveguide photodiode structure

\section{Modeling}

\subsection{Transit Time Effects}

The continuity equations for holes and electrons in the absorption region may be written as:

$$
\begin{aligned}
\frac{\partial p(x, y, t)}{\partial t}= & -\frac{p(x, y, t)}{\tau_{p}}+\alpha g_{0} e^{-\alpha x} \delta(t)+ \\
& +D_{p} \frac{\partial^{2} p(x, y, t)}{\partial x^{2}}-v_{p} \frac{\partial p(x, y, t)}{\partial y} \\
\frac{\partial n(x, y, t)}{\partial t}= & -\frac{n(x, y, t)}{\tau_{n}}+\alpha g_{0} e^{-\alpha x} \delta(t)+ \\
& +D_{n} \frac{\partial^{2} n(x, y, t)}{\partial x^{2}}+v_{n} \frac{\partial n(x, y, t)}{\partial y}
\end{aligned}
$$

where $v_{p}$ and $v_{n}, D_{p}$ and $D_{n}, \tau_{p}$ and $\tau_{n}$ are the hole and electron saturation velocities, diffusion coefficients and lifetimes respectively. $\alpha$ is the absorption coefficient, $g_{0}$ the incident photon flux at $\mathrm{x}=0$ and $\delta(t)$ the unit impulse function.

By assuming that all the photo-generated carriers, due to the electric field, are instantly carried away in the ydirection, the hole and electron density in the frequency domain, $P(x, y)$ and $N(x, y)$, may be expressed by

$$
\begin{aligned}
& P(x, y)=\frac{\alpha g_{0} e^{-\alpha x}}{j \omega+1 / \tau_{p}}\left[1-e^{-\left(j \omega+1 / \tau_{p}\right) y / v_{p}}\right] \\
& \left.N(x, y)=\frac{\alpha g_{0} e^{-\alpha x}}{j \omega+1 / \tau_{n}}\left\{1-e^{-\left(j \omega+1 / \tau_{n}\right)\left[(y-d) / v_{n}\right.}\right]\right\}
\end{aligned}
$$

The current density in the frequency domain depends on the electron and hole density and may be obtained from:

$$
J(x)=\frac{q}{d} \int_{0}^{d}\left[N(x, y) v_{n}+P(x, y) v_{p}\right] d y
$$

The frequency response of the WGPD structure shown in Figure 1 is obtained by considering the transit time, capacitive and inductive effects.

The total photocurrent density in the photodetector may be written as:

$$
I(\omega)=\frac{1}{L} \int_{0}^{L} J(x) d x
$$

\subsection{Capacitive and Inductive Effects}

The capacitive and inductive effects may be included in the model by using the small signal equivalent circuit for the pin structure, Figure 2 (El-Batawy \& Deen, 2005). 


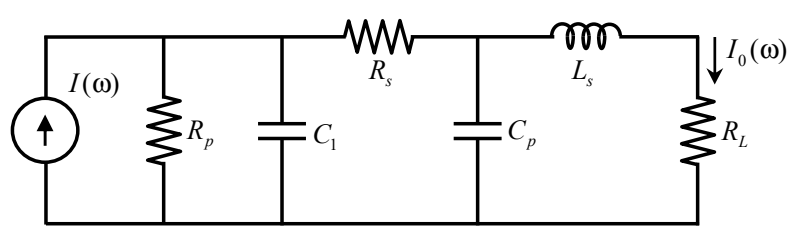

Figure 2. Small signal equivalent circuit for the pin structure

In the circuit $C_{1}$ represents the capacitance involving the absorption layer, $C_{p}$ is the parasitic capacitance, $L_{S}$ is the parasitic inductance and $R_{L}$ is the load resistance. The leakage and series resistance are represented by $R_{p}$ and $R_{S}$ respectively.

$C_{l}$ may be written as

$$
C_{1}=\varepsilon_{a} \frac{A}{d}
$$

with $A$ being the cross-sectional area of the device and $\varepsilon_{\mathrm{a}}$ the electric permittivity of the semiconductor material used in the absorption region.

For this circuit, the transfer function, neglecting $R_{p}$, may be written as:

$$
\begin{array}{r}
H_{R L C}(\omega)=1 /\left\{1-\omega^{2}\left[L_{S}\left(C_{1}+C_{p}\right)+C_{1} C_{p} R_{S} R_{L}\right]+\right. \\
\left.+j \omega\left[C_{1}\left(R_{L}+R_{S}\right)+C_{p} R_{L}-\omega^{2} C_{1} C_{p} R_{S} L_{S}\right]\right\}
\end{array}
$$

The frequency response of the device may be computed in the frequency domain by performing the product between the frequency response due to the transit time effects and the frequency response associated with the capacitive and inductive effects:

$$
H(\omega)=\frac{I(\omega)}{I_{0}} \times H_{R L C}(\omega)
$$

where $I_{0}$ is the low frequency response of the photodiode associated with the transit time effects.

\section{Results and Discussion}

The density of electrons and holes in the frequency domain is represented in Figure 3 and 4 respectively. The device is assumed to have: $d=0.6 \mu \mathrm{m}, L=2 \mu \mathrm{m}$. The relative electric permittivity for the absorption region is 14.1 and its absorption coefficient $\alpha=0.68 \mu \mathrm{m}^{-1}$ for $\lambda=1.55 \mu \mathrm{m}$. The electron and hole saturation velocities, $v_{n}$ and $v_{p}$, are assumed to be $6.5 \times 10^{4} \mathrm{~m} / \mathrm{s}$ and $4.8 \times 10^{4} \mathrm{~m} / \mathrm{s}$ respectively and the electron and hole lifetime $\tau_{\mathrm{n}}=\tau_{\mathrm{p}}=20 \mu \mathrm{s}$ (Das $\&$ Deen, 2005). The density is in arbitrary units, but they can be used for comparison purposes because they are identically scaled.

The electrons move along the negative y direction, and thus, the carrier density has a tendency to rise along that direction, Figure 3. The signal frequency also plays a significant role in the position dependence of the electron density, as shown in Figure 3(a) and Figure 3(b). For higher frequencies, the high density positions are further away from the positive electrode $(\mathrm{y}=0)$, indicating a lagging of carriers. Similarly the hole density has a tendency to rise along the positive y direction, Figure 4. At higher frequencies the peak of the hole density lags further behind the negative electrode $(\mathrm{y}=\mathrm{d})$.

The photocurrent density is dependent on $\mathrm{x}$ and is represented in Figure 5, as a function of frequency, for $\mathrm{x}=0$; 0.8 and $1.6 \mu \mathrm{m}$. It is seen that higher current densities are obtained at $\mathrm{x}=0$, where the electron-hole photo-generated pairs density is larger. An increase of frequency decreases the current density. 

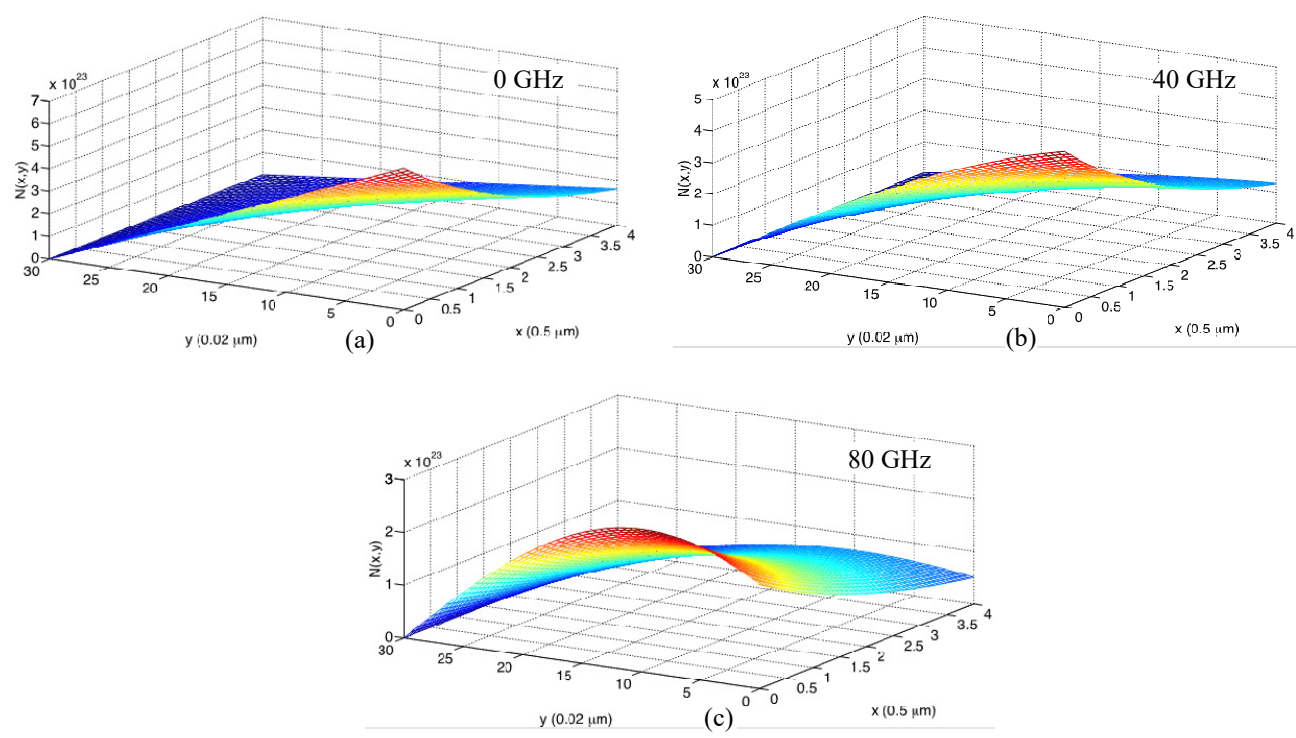

Figure 3. Distribution of electron density at several frequencies. The y scale is multiplied by 0.02 and the $x$ scale is multiplied by 0.5 to get the corresponding lengths in $\mu \mathrm{m}$

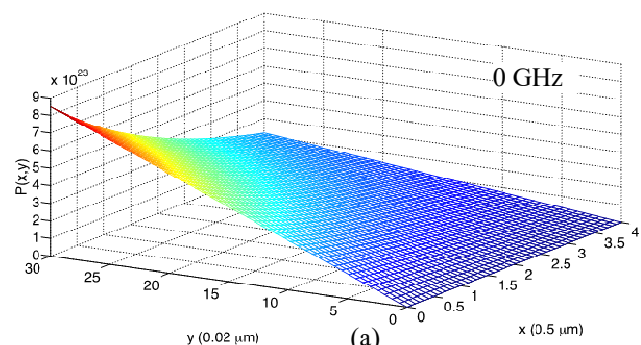

(a)

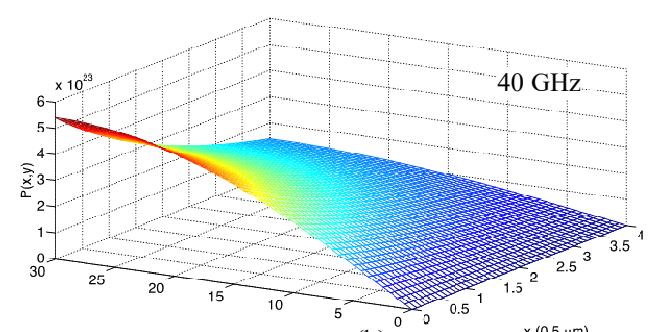

(b)

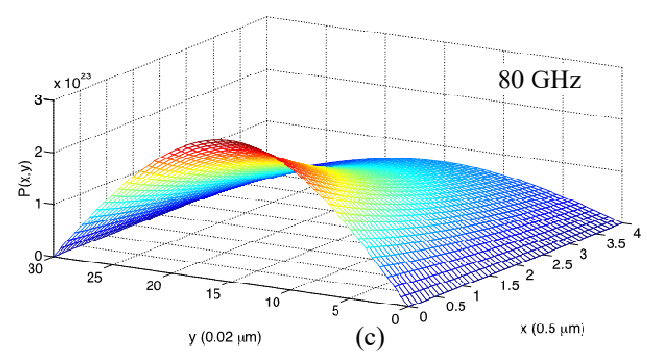

Figure 4. Distribution of hole density at several frequencies

The bandwidth as a function of the absorption region width is shown in Figure 6 where the capacitive and inductive effects have been included. It is assumed that the length $L$ and width $W$ of the device are $20 \mu \mathrm{m}$ and $6 \mu \mathrm{m}$ respectively and that $R_{S}=6 \Omega, R_{L}=50 \Omega$ and $C_{p}=6 \mathrm{fF}$. When the capacitive effects are included the maximum bandwidth is $46 \mathrm{GHz}$ for a thickness $d=0.41 \mu \mathrm{m}$, which agree with other published results for similar structures (Umbach et al., 1996). 


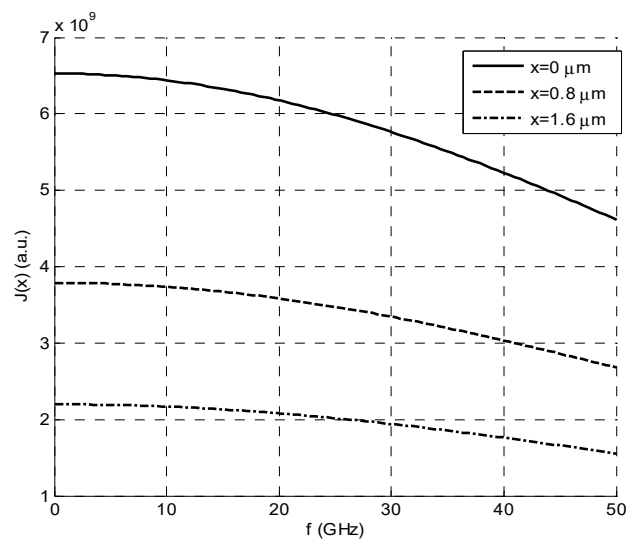

Figure 5. Current density as a function of frequency for several values of $\mathrm{x}$

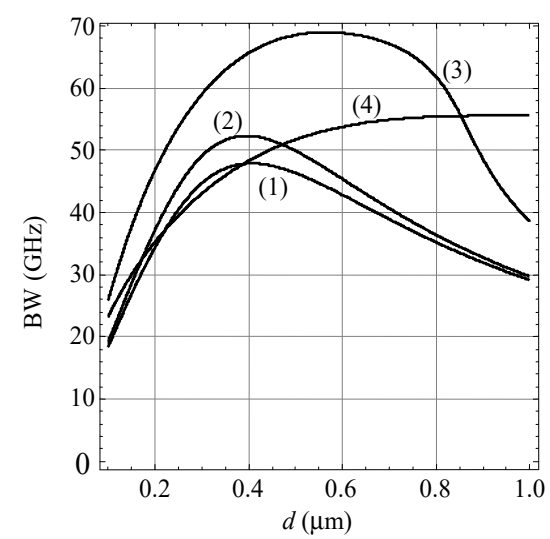

Figure 6. Bandwidth versus $d$ for $\mathrm{L}_{\mathrm{S}}=$ (1) 0 ; (2) $0.02 \mathrm{nH}$; (3) $0.2 \mathrm{nH}$; (4) $0.4 \mathrm{nH}$

Decreasing the area of the metallic contact, the series resistance and the parasitic capacitance, higher bandwidths may be obtained. By including a parasitic inductance it is possible to cancel in part the capacitive effects. With an inductance $L_{S}=0.2 \mathrm{nH}$ the bandwidth is seen to reach a maximum of about $70 \mathrm{GHz}$, for thicknesses in the range 0.5-0.6 $\mu \mathrm{m}$. Figure 7 shows the bandwidth as a function of $L_{S}$ for several values of the absorption region thickness. It is seen that the frequency response may be optimized by the proper choice of $L_{S}$ for each value of $d$, e.g., when $d=0.1,0.6,0.8$ and $1 \mu \mathrm{m}$ the maximum bandwidth is obtained for $L_{S}=0.25,0.2,0.25$ and $0.35 \mathrm{nH}$ and it takes the value $26,68,64$ and $60 \mathrm{GHz}$ respectively. It is also important to notice that, except for the device with $d=0.1 \mu \mathrm{m}$, the inductive effects always lead to an increase of the device's bandwidth.

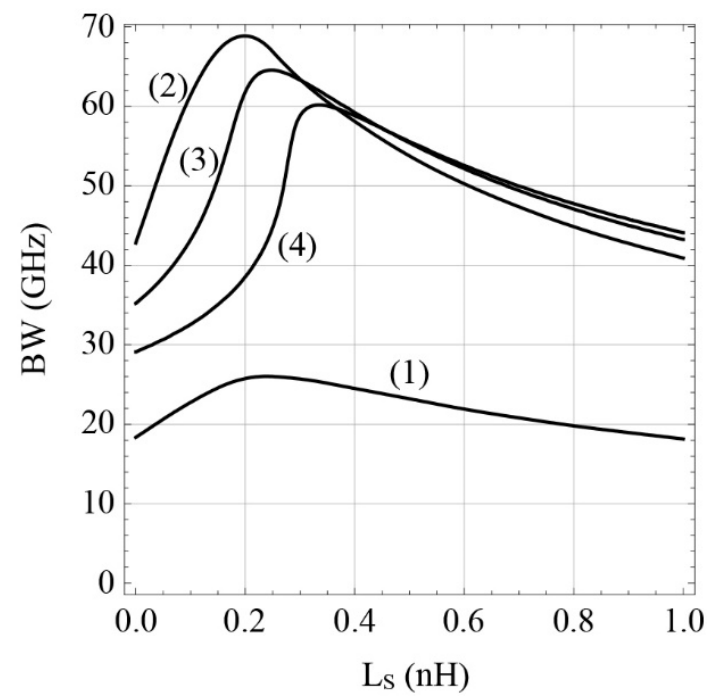

Figure 7. Bandwidth versus $\mathrm{L}_{\mathrm{S}}$ for $d=$ (1) $0.1 \mu \mathrm{m}$; (2) $0.6 \mu \mathrm{m}$; (3) $0.8 \mu \mathrm{m}$; (4) $1 \mu \mathrm{m}$

Therefore, the bandwidth optimization procedure has to take into account the photodetector dimensions, parasitic resistances and capacitances and the load resistance in the choice of the series inductance.

\section{Conclusions}

Waveguide photodetectors are more versatile than the conventional pin photodiodes because the bandwidth and the quantum efficiency may be controlled almost independently. This paper investigates the frequency response of a waveguide photodetector. By decreasing the thickness of the absorption region the bandwidth increases and reaches a maximum for $d=0.4 \mu \mathrm{m}$. The existence of this maximum can be related to the capacitive effects which are dominant for smaller thicknesses. Above $0.4 \mu \mathrm{m}$ the bandwidth is mainly dependent on the transit time effects. The inclusion of a small parasitic inductance has an important effect on the bandwidth. It is shown that the properly optimized device may double its bandwidth. 


\section{Acknowledgements}

This work was support by FCT, under IT, Project No. UID/EEA/50008/2013.

This work was sponsored by Instituto deTelecomunicações, pólo de Lisboa, project P186.

\section{References}

Bowers, J., \& Burrus, C. (1987). Ultrawide-band long-wavelength pin photodetectors. Journal of Lightwave Technology, 5(10), 1339-1350. https://doi.org/10.1109/JLT.1987.1075419

Das, N. R., \& Deen, M. J. (2005). A model for the performance analysis and design of waveguide pin photodetectors. IEEE Transactions on Electron Devices, 52(4), 465-472. https://doi.org/10.1109/TED.2005.845152

El-Batawy, Y. M., \& Deen, M. J. (2005). Analysis, circuit modeling, and optimization of mushroom waveguide photodetector (mushroom-WGPD). Journal of lightwave technology, 23(1), 423. https://doi.org/10.1109/JLT.2004.834483

Fernandes, C. C., \& Pereira, J. T. (2011, April). Bandwidth modeling and optimization of PIN photodiodes. In EUROCON-International Conference on Computer as a Tool (EUROCON), 2011 IEEE (pp. 1-4). IEEE.

Kato, K., Hata, S., Kawano, K., Yoshida, J., \& Kozen, A. (1992). A high-efficiency 50 GHz InGaAs multimode waveguide photodetector. IEEE Journal of Quantum Electronics, 28(12), 2728-2735. https://doi.org/10.1109/3.166466

Pearsall, T. (1980). Ga ${ }_{0.47} \mathrm{In}_{0.53}$ As: a ternary semiconductor for photodetector applications. IEEE Journal of Quantum Electronics, 16(7), 709-720. https://doi.org/10.1109/JQE.1980.1070557

Umbach, A., Trommer, D., Mekonnen, G. G., Ebert, W., \& Unterborsch, G. (1996). Waveguide integrated 1.55/spl $\mathrm{mu} / \mathrm{m}$ photodetector with $45 \mathrm{GHz}$ bandwidth. Electronics Letters, 32(23), 2143-2145. https://doi.org/10.1049/el:19961425

\section{Copyrights}

Copyright for this article is retained by the author(s), with first publication rights granted to the journal.

This is an open-access article distributed under the terms and conditions of the Creative Commons Attribution license (http://creativecommons.org/licenses/by/4.0/). 